\title{
Alzheimer Patient Tracking System in Indoor Wireless Environment
}

\author{
Prima Kristalina \\ Achmad Ilham Imanuddin \\ Mike Yuliana \\ Aries Pratiarso \\ I Gede Puja Astawa
}

Electronic Engineering Polytechnic Institute of Surabaya, Indonesia

Doi: 10.19044/esj.2017.v13n33p327 URL:http://dx.doi.org/10.19044/esj.2017.v13n33p327

\begin{abstract}
Alzheimer's disease is a disease of the nerves that are irreversible, resulting in memory impairment. This condition resulted in Alzheimer's patients easily lost because they forget the existence. In this research, we designed a tracking system for Alzheimer's patients in a hospital environment, incorporating Kalman method to estimate the position of the patient. As known Received Signal Strength Indicator value is strongly influenced by environmental conditions that lead to the acquisition of position estimation is inaccurate. From the test results showed that the optimal Kalman estimated value obtained when the value of $\mathrm{R}=0: 01$ and $\mathrm{Q}$ $=0.1$ with the average percentage of error only $7.01 \%$ of the actual patient position. The test results with various data variations also indicate the reliability of the Kalman method, because of the average estimated position approach the actual patient position.
\end{abstract}

Keywords: Alzheimer, tracking, Kalman, position, RSSI

\section{Introduction}

Today there are many cases of Alzheimer's disease in the world. Alzheimer's disease is a disease of the nerves that are irreversible, causing damage to memory, judgment, decision making, and physical orientation (Chancellor et. al, 2014). Many people with Alzheimer's who have received treatment with hospitalized. But often these patients are unknown because they are often lost and forgotten its existence, although in a hospital room or environment. This happens because the supervision of Alzheimer's sufferers still uses manual control. 
The technology that could be used to overcome these problems is the use of Wi-Fi and Bluetooth technology. The use of these two technologies is very helpful for tracking the position of objects in the indoor environment. The accuracy of position tracking results is strongly influenced by noise caused by indoor environmental conditions with various obstacles (Chauhan et. al, 2014). This inaccurate measurement result can be a serious problem in a tracking system design.

In this research, we propose a tracking system for Alzheimer's patients, incorporating Kalman method for estimating the position so that it can prevent the loss of Alzheimer's patients. Kalman filter is a reliable estimator and does not consider the noise that occurs in an environment in detail. The system is built using a technology that is iBeacon and Raspberry $\mathrm{Pi}$ mini PC. IBeacon is Bluetooth Low Energy (BLE) technology. IBeacon module is much smaller and can be planted on devices that have a limited size and space. This module will send an RSSI parameter that can be used to determine the distance between patients with a mini PC that serves as a receiver. Due to the amount of noise, it is possible that the position obtained does not match the actual position, so it is necessary to use the Kalman method to estimate the position obtained.

\section{Alzheimer}

Alzheimer's is the loss of intellectual and social abilities severe enough to affect daily activities. In Alzheimer's disease, brain tissue health has decreased, causing a decline in memory and mental abilities. Alzheimer's is not a contagious disease; it is a kind of syndrome with apoptosis of brain cells at about the same time, so the brain appears to shrivel and shrink. Alzheimer's is also said to be a synonymous disease with old people. Alzheimer's is not part of the normal aging process, but the risk increases with age. Five percent of people aged between 65-74 years have Alzheimer's disease, and almost 50 percent of people older than 85 years have Alzheimer's disease.

\section{Target Tracking}

There are 3 stages of tracking on a wireless network that includes taking measurement data between nodes, converting measurement data to distance parameters and position estimation process (Pratiarso et. al, 2015). From these three steps, we use a range-based method to get strong signal data received from other nodes, convert the signal strength value to distance using log-normal shadowing modeling and calculate the patient position estimation by Kalman Filter method. 


\section{Measurement Data Collection}

Measurement data is a parameter that will be used to estimate the distance between nodes. This data consists of various types, such as using signal strength received from another node (Received Signal Strength Indicator / RSSI), Time Difference of Arrival (TDOA) or measuring signal arrival angle to the receiver (Angle of Arrival / AOA). The RSSI technique measures the signal strength received from the receiving device equipped with one of the wireless communication modules, such as Access Point, Zigbee, Bluetooth, and RF. TDOA techniques used for devices that are equipped with the ultrasonic module and AOA techniques applied to devices equipped with the antenna array. The magnitude of the measured data at RSSI technique using decibels and the magnitude is inversely proportional to the distance between a sending device and the receiving signal strength (Martin et. al, 2014). The farther the distance, the smaller the value of the received signal strength. Signal strength collection mechanism is shown in Fig. 1.

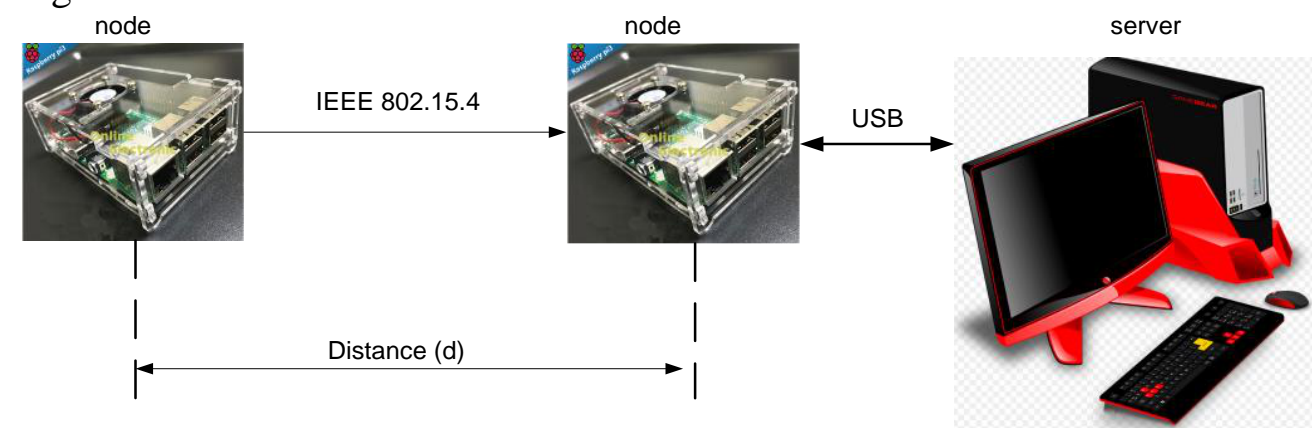

Figure 1 Signal strength collection mechanism.

\section{Log-normal Shadowing Channel Modeling}

At the RSSI algorithms, signal strength is very important in determining the estimated distance between the nodes. In fact, many factors can affect the signal strength received by among others multipath fading, shadowing, antenna effect, and the effect of transmission equipment itself. Therefore, it needs a channel model to reduce the propagation loss as shown in Fig. 2. Path loss model is a generic and development model of the Friis Free space model. It is used to predict propagation losses for various environments. Meanwhile, Friis free space models are restricted to an area that obviously contained a barrier between transmitter and receiver (Okumbor et. al, 2014). 


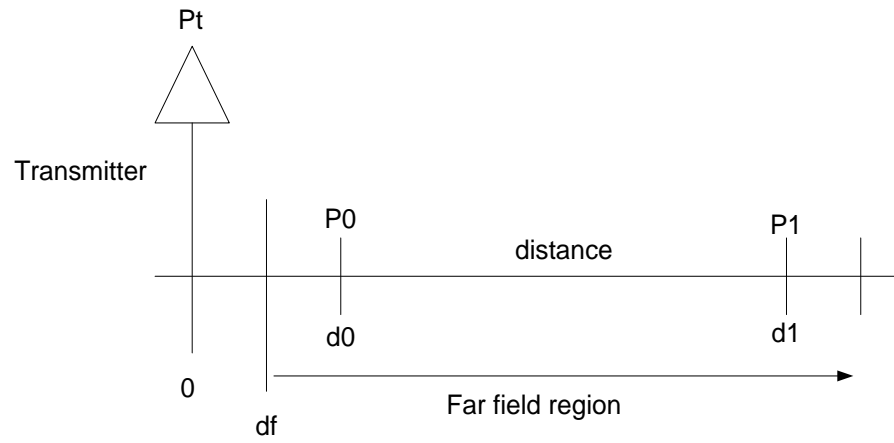

Figure 2 Log-normal Shadowing Channel Modeling.

In areas far from the transmitter $\left(d \geq d_{f}\right)$, if the $\operatorname{PL}\left(d_{0}\right)$ is the value of path loss at the distance $\mathrm{d}_{0}$ from the transmitter, then the path loss at a certain distance $d>d_{0}$ then its decline can be seen in equation (4).

$$
P_{L}=P_{T X}-P_{R X}
$$

By using reference 1 meter as $\mathrm{d}_{0}$, then the $n$ value obtained from (5) and (6).

$$
\begin{gathered}
P_{L}=P_{L 0}+10 \cdot n \cdot \log \frac{d}{d_{0}}+X_{\sigma} \\
-P_{R X}=-P_{R X 0}+10 \cdot n \cdot \log \frac{d}{d_{0}}+X_{\sigma}
\end{gathered}
$$

By using the value of $n$ from (6), then at (7) obtained path loss coefficient for measurement conditions at the same angle.

$$
n=\frac{P_{R X 0}-P_{R X}}{10 \log \frac{d}{d_{0}}}-X_{\sigma}
$$

$X_{\sigma}$ is a zero-mean variable Gaussian distributed random with standard deviation $\sigma$. This is only used when there is a shadowing effect (Nebe, 2014). If there is no shadowing effect, then this variable is zero. Estimated distance between the node $n$ has been obtained from (7) is shown by (8). Table 1 shows the path loss exponent for different environments (Kristalina et. al, 2014) .

$$
d=d_{0} 10^{\left(\frac{P_{R_{X 0}}-P_{R_{X}}-X_{\sigma}}{10 n}\right)}
$$

Table 1 Path loss exponent for a different environment.

Environment

Free space

Urban area cellular radio

Shadowed urban cellular radio

In building Line-of-sight

Obstructed in building
Path Loss Exponent, $\mathrm{n}$

$$
2
$$

2.7 to 3.5

3 to 5

1.6 to 1.8

4 to 6

\section{Estimation of Node Position with Kalman Filter}

The time and measurement update scheme of the Kalman Filter method can be seen in Fig. 3. $\hat{x}_{k}$ is a priori estimation state (previous state) 
for the time step $\mathrm{k}$, obtained by multiplying the filter parameter $A$ (system state) with $\hat{x}_{k}$, namely the estimated posteriori state (next state) for the time step $\mathrm{k}-1$. The result is then added to the filter $B$ (input gain) parameter multiplied by the input control $u_{k}$. $\hat{P}_{k}$ is priori estimate error covariance, while $P_{k}$ is posteriori estimate error covariance. In practice the process noise covariance, $Q$ and measurement noise covariance, $R$ can be changed each time step or after each measurement. $K_{k}$ is Kalman gain based on filter parameter $H$ (output gain) and $z_{k}$ is actual measurement. The final result of Kalman filter estimation is $x_{k}$ (Ali et. al, 2014).

The parameters of the Kalman Filter algorithm include the state system $x_{k-1}$ and the matched covariance matrix $P_{k-1}$, and the noise parameters $R$ and $Q$. These noise parameters can be estimated every iteration of the Kalman Filter, but can also be of fixed value. In rows 1 and row 2 , the prediction $\hat{x}_{k}$ and covariance $\hat{P}_{k}$ are based on state system $x_{k-1}$ and previous input control $u_{k-1}$. In row 3 Kalman Gain is calculated. Finally, rows 4 and 5 produce the new $x_{k}$ state system and covariance of the new $P_{k}$ matrix calculated using the previous state and Kalman Gain.

\section{$\underline{\text { Time Update }}$}

State Prediction

$\hat{x}_{k}=A x_{k-1}+B u_{k-1}$

Covariance Error Prediction

$\hat{P}_{k}=A P_{k-1} A^{T}+Q$

Measurement Update

Calculating Kalman Gain

$K_{k}=\hat{P}_{k} H^{T}\left(H \hat{P}_{k} H^{T}+R\right)^{-1}$

Estimation Update $z_{k}$

$x_{k}=\hat{x}_{k}+K_{k}\left(z_{k}-H \hat{x}_{k}\right)$

Covariance Error Update

$P_{k}=\left(1-K_{k} H\right) \hat{P}_{k}$

Figure 3 The time and measurement update scheme of the Kalman Filter method. 


\section{System Design}

In this research will be designed a tracking system for the position of Alzheimer's patients using iBeacon and Raspberry Pi devices. Block diagram of the overall system can be seen in Fig.4. Each Alzheimer's patient will be equipped with an iBeacon device to send ID and signal strength to Raspberry Pi. Signal strength data will be sent to the server to convert to distance. What is meant by the distance here is the distance from the position of the patient to the Raspberry Pi. Due to the amount of noise, it is possible that the distance data obtained does not match the actual patient position, so it is necessary to use the Kalman method to estimate the distance data obtained.

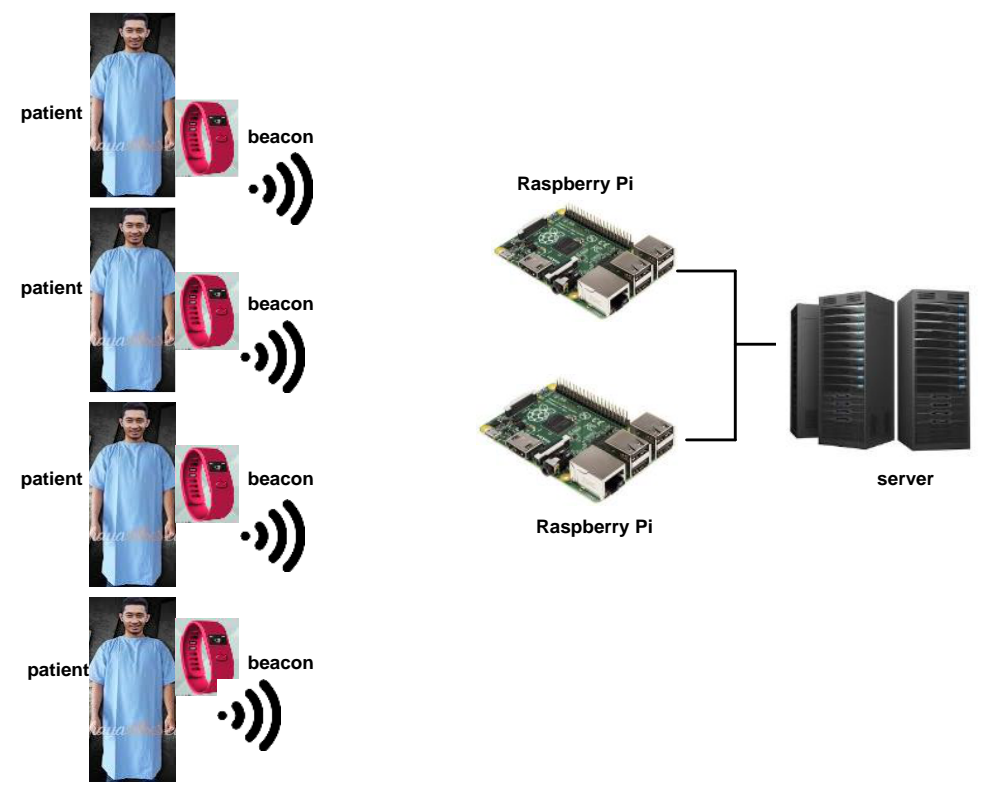

Figure 4 Block diagram of the system.

There are three steps that must be performed in this research, where the step include:

1. Node deployment scenario

2. Collecting RSSI data for path loss coefficients

3. Converting RSSI to distance

4. Estimating the patient position using the Kalman method

\section{Node Deployment Scenario}

Determining the distribution of nodes will be adapted to the indoor environment at the Dr. M. Soewandhie hospital. Fig.5. is an indoor environmental map of Dr. M. Soewandhie hospital at 2nd floor. In this research will be distributed 10 Raspberry Pi (orange color) with a distance 
of 15-20 meters at a predetermined position and 2 iBeacon devices (purple color) in a changing position as seen in Fig. 6

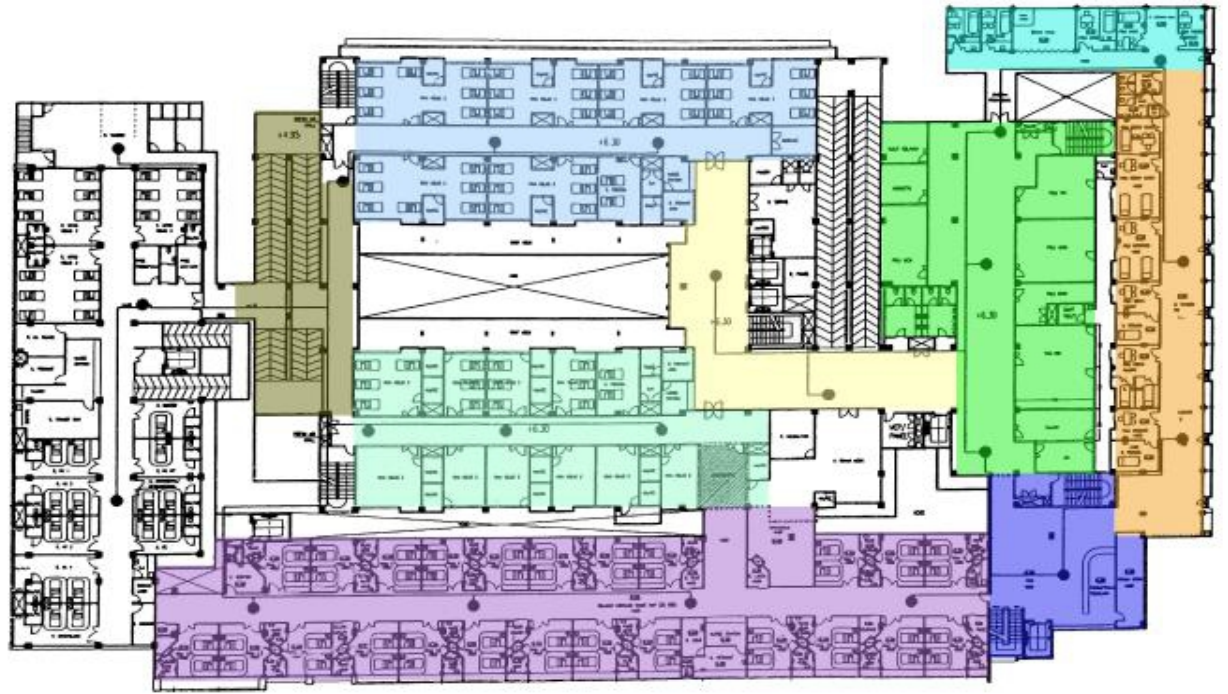

Figure 5 Indoor environment at the Dr. M. Soewandhie hospital.

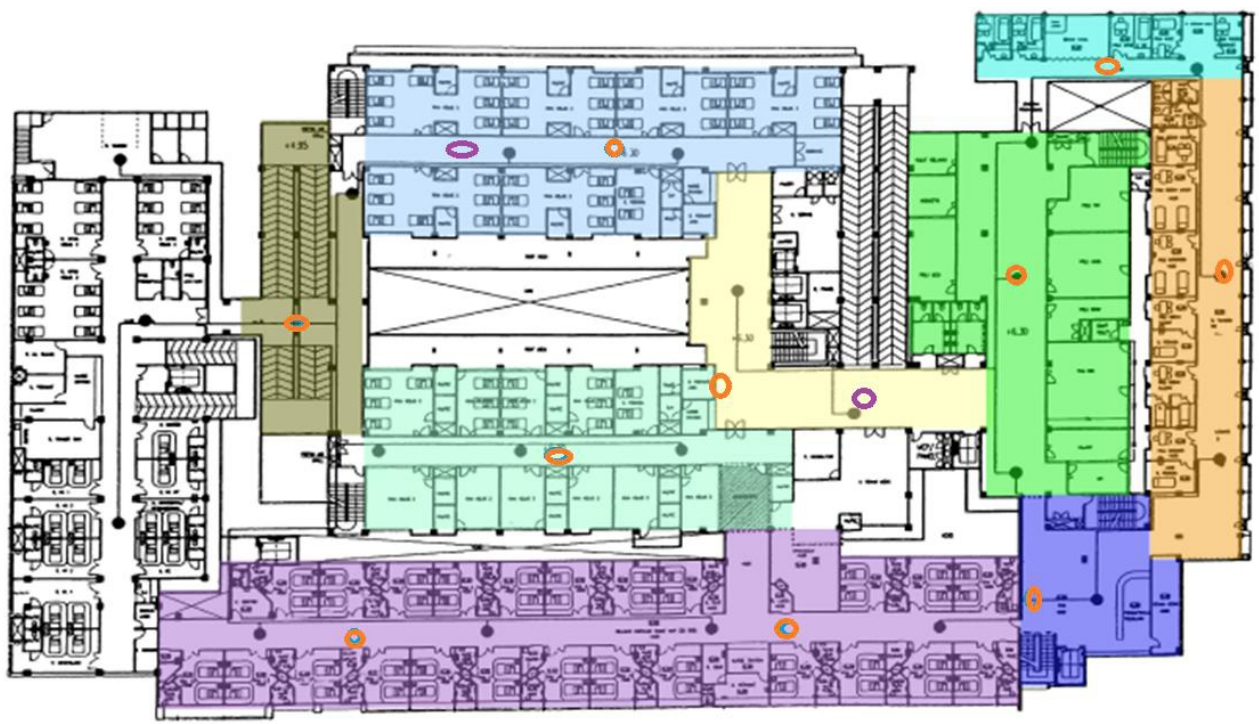

Figure 6 Node deployment scenario.

\section{Collecting RSSI Data for Path Loss Coefficients}

There are 3 stages to obtain the path loss coefficient, wherein the third stage includes signal strength measurement of the uniformly spread to get a reference power, signal strength measurement with rising distance of 2 meters, calculating the average value of the coefficient of path loss. At the signal strength measurement of the uniformly spread, field observations will be divided into several areas. Each area is measured by the distance between 
the nodes as far as 1 meter. In the second stage, the receiver node (Raspberry $\mathrm{Pi}$ ) is placed at one end of the room and the transmitter node (iBeacon) is placed 2 meters away from the Rx node. The final result is the average path loss coefficient values that will be used to estimate the distance to the raspberry $\mathrm{Pi}$ iBeacon.

\section{Converting RSSI to Distance}

There are two stages used to convert RSSI to a distance, which includes:

1. Getting a signal strength value $P_{R_{x}}$ from beacon.

2. Converting strength signal into distance by using parameter $P_{R_{x 0}}$ and $n$.

\section{Estimating the Patient Position using The Kalman Method}

In this section, the distance data obtained from the RSSI conversion process will be estimated through two phases, namely the prediction and correction phase. The prediction phase estimates the state in the previous distance data while the correction phase incorporates new measurement data with predictive data to obtain new distance data approach to the actual patient position.

\section{Performance Evaluation System}

Performance of the system will be evaluated using 3 tests, which includes testing the accuracy of the RSSI conversion distance data, testing the patient position estimation accuracy with the Kalman filter, as well as testing the computational time of the Kalman Filter.

\section{Testing the Accuracy of the RSSI Conversion Distance Data}

From the measurement results uniformly spread of signal strength and signal strength measurement with rising distance of 2 meters obtained the value $n$ of 1.26. Table 1 show that the environment within the LOS building should have $n$ value between 1.6 up 1.8. So it can be said that the value of $n$ measurement results has an error value of $13 \%$. Table 2 shows that there are some estimate values $(d)$ that approach to the actual value with a percentage error of $3 \%$. But at an actual position of 25 meters to 74 meters, has a very large percentage error that is more than $100 \%$. This occurs because of the surrounding environmental conditions that affect the strength signal value that received from iBeacon. 


\begin{tabular}{|c|c|c|c|c|c|}
\hline $\begin{array}{l}\text { Actual } \\
\text { Position } \\
\text { (meter) }\end{array}$ & RSSI & $\begin{array}{c}\mathrm{d} \\
\text { (meter) }\end{array}$ & $\begin{array}{l}\text { Actual } \\
\text { Position } \\
\text { (meter) }\end{array}$ & RSSI & $\begin{array}{c}\mathrm{d} \\
\text { (meter) }\end{array}$ \\
\hline 2 & -57.04 & 1.311000 & 40 & -77.8 & 56.7839 \\
\hline 4 & -58.22 & 1.624000 & 42 & -84.38 & 187.4671 \\
\hline 6 & -65.04 & 5.600000 & 44 & -85.56 & 232.2439 \\
\hline 8 & -66.34 & 7.093099 & 46 & -84.16 & 180.1285 \\
\hline 10 & -65.1 & 5.663529 & 48 & -81.9 & 119.5163 \\
\hline 12 & -73.94 & 28.18008 & 50 & -81.66 & 114.4216 \\
\hline 14 & -74.76 & 32.70263 & 52 & -77.48 & 53.57963 \\
\hline 16 & -76.08 & 41.55641 & 54 & -79.24 & 73.74627 \\
\hline 18 & -71.66 & 18.62989 & 56 & -78.2 & 61.06004 \\
\hline 20 & -65.22 & 5.788242 & 58 & -73.52 & 26.11162 \\
\hline 22 & -68.1 & 9.762845 & 60 & -71.14 & 16.95192 \\
\hline 24 & -71.18 & 17.07545 & 62 & -70.36 & 14.71403 \\
\hline 26 & -74.36 & 30.41241 & 64 & -75.98 & 40.80891 \\
\hline 28 & -78.72 & 67.10403 & 66 & -73.78 & 27.37345 \\
\hline 30 & -81.2 & 105.2559 & 68 & -75.5 & 37.40389 \\
\hline 32 & -77.94 & 58.24536 & 70 & -72.62 & 22.17619 \\
\hline 34 & -79.28 & 74.28365 & 72 & -74.44 & 30.85725 \\
\hline 36 & -77.4 & 52.80722 & 74 & -76.3 & 43.24945 \\
\hline 38 & -80.5 & 92.69698 & & & \\
\hline
\end{tabular}

\section{Testing the Patient Position Estimation Accuracy with The Kalman Filter}

There are several parameters that will be used in testing this Kalman filter method, the first is the covariance of process noise $(Q)$, the second is the measurement covariance noise $(R)$. The values of both parameters will be varied from $10^{-1}, 10^{-2}, 10^{-3}, 10^{-4}$ and $10^{-5}$. This variation is done to obtain optimal estimation of the given noise.

Table 3 Examples of patient position estimation by Kalman (6 meters)

\begin{tabular}{|c|c|c|c|c|c|c|c|c|c|c|c|}
\hline & \multicolumn{10}{|c|}{ Covariance of Measurement Noise $(R)$} \\
\hline & & \multicolumn{2}{|c|}{$10^{-5}$} & \multicolumn{2}{|c|}{$10^{-4}$} & \multicolumn{2}{|c|}{$10^{-3}$} & \multicolumn{2}{|c|}{$10^{-2}$} & \multicolumn{2}{|c|}{$10^{-1}$} \\
\hline & & d & $\begin{array}{c}\% \\
\text { Err }\end{array}$ & d & $\begin{array}{c}\% \\
\text { Err }\end{array}$ & d & $\begin{array}{c}\% \\
\text { Err }\end{array}$ & d & $\begin{array}{c}\% \\
\text { Err }\end{array}$ & d & $\% \mathrm{Err}$ \\
\hline \multirow{5}{*}{ 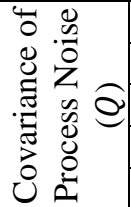 } & $10^{-5}$ & 5.73 & $4 \%$ & 5.92 & $1 \%$ & 3.6 & $40 \%$ & 2.92 & $51 \%$ & 2.79 & $54 \%$ \\
\hline & $10^{-4}$ & 6.27 & $4 \%$ & 5.73 & $4 \%$ & 5.92 & $1 \%$ & 3.6 & $40 \%$ & 2.91 & $52 \%$ \\
\hline & $10^{-3}$ & 6.23 & $4 \%$ & 6.27 & $4 \%$ & 5.73 & $4 \%$ & 5.92 & $1 \%$ & 3.6 & $40 \%$ \\
\hline & $10^{-2}$ & 6.22 & $4 \%$ & 6.23 & $4 \%$ & 6.27 & $4 \%$ & 5.73 & $4 \%$ & 5.92 & $1 \%$ \\
\hline & $10^{-1}$ & 6.22 & $4 \%$ & 6.22 & $4 \%$ & 6.23 & $4 \%$ & 6.27 & $4 \%$ & 5.73 & $4 \%$ \\
\hline
\end{tabular}

Table 3 shows that the percentage of the smallest error (Err) obtained at a patient position estimation of 6 meters i.e. $1 \%$ on the parameter $\mathrm{R}=0.1$ and $\mathrm{Q}=0.01$, respectively. If both values of these parameters are applied to a patient position estimation of 2 meters to 74 meters, the largest error percentage obtained up to $180 \%$. The most optimal estimates result obtained 
when the value of $\mathrm{R}=0.01$ and $\mathrm{Q}=0.1$ with the average error measurement is $7.01 \%$ of the actual patient position. While the worst estimate results obtained when $\mathrm{R}=0.01$ and $\mathrm{Q}=0.00001$ with the average value up to $50 \%$.

To test the reliability of Kalman, then the estimate patient position will be performed again using a different data range of each patient position obtained from previous measurements as shown in Table 4. Fig.7 shows the reliability of Kalman where the difference in range of data 1 time (variant 1), 2 times (variant 2), and 3 times (variant 3) of the measurement data, the patient position obtained is not much different from real data, with the largest percentage error obtained up to $46 \%$.

Table 4 Range of data from each actual patient position.

\begin{tabular}{|c|c|c|c|c|c|c|c|c|c|}
\hline Actual & $\mathrm{Mi}$ & Media & $\mathrm{Ma}$ & $\begin{array}{l}\text { Rang } \\
\mathrm{e}\end{array}$ & Actual & $\mathrm{Mi}$ & Media & $\mathrm{Ma}$ & $\begin{array}{l}\text { Rang } \\
\text { e }\end{array}$ \\
\hline Position & $\mathrm{n}$ & $\mathrm{n}$ & $\mathrm{x}$ & $\begin{array}{l}\text { Valu } \\
\mathrm{e}\end{array}$ & Position & $\mathrm{n}$ & $\mathrm{n}$ & $\mathrm{X}$ & $\begin{array}{l}\text { Valu } \\
\mathrm{e}\end{array}$ \\
\hline 2 & -69 & -60.5 & -46 & 23 & 40 & -94 & -80 & -66 & 28 \\
\hline 4 & -69 & -59 & -49 & 20 & 42 & $\begin{array}{l}- \\
100\end{array}$ & -87 & -74 & 26 \\
\hline 6 & -84 & -67 & -50 & 34 & 44 & $\begin{array}{l}- \\
101\end{array}$ & -88.5 & -76 & 25 \\
\hline 8 & -80 & -68 & -56 & 24 & 46 & -96 & -84 & -72 & 24 \\
\hline 10 & -77 & -66 & -55 & 22 & 48 & -96 & -83 & -70 & 26 \\
\hline 12 & -87 & -73 & -59 & 28 & 50 & -95 & -83 & -71 & 24 \\
\hline 14 & -94 & -77.5 & -61 & 33 & 52 & -95 & -81 & -67 & 28 \\
\hline 16 & -98 & -80 & -62 & 36 & 54 & -92 & -80 & -68 & 24 \\
\hline 18 & -89 & -73.5 & -58 & 31 & 56 & $\begin{array}{l}- \\
101\end{array}$ & -83 & -65 & 36 \\
\hline 20 & -82 & -69.5 & -57 & 25 & 58 & -84 & -74 & -64 & 20 \\
\hline 22 & -79 & -68.5 & -58 & 21 & 60 & -88 & -74.5 & -61 & 27 \\
\hline 24 & -82 & -71 & -60 & 22 & 62 & -82 & -72 & -62 & 20 \\
\hline 26 & -95 & -78.5 & -62 & 33 & 64 & -85 & -75 & -65 & 20 \\
\hline 28 & -91 & -80 & -69 & 22 & 66 & -92 & -78 & -64 & 28 \\
\hline 30 & -99 & -83.5 & -68 & 31 & 68 & -89 & -77.5 & -66 & 23 \\
\hline 32 & -93 & -79 & -65 & 28 & 70 & -84 & -73.5 & -63 & 21 \\
\hline 34 & -94 & -82 & -70 & 24 & 72 & -88 & -76.5 & -65 & 23 \\
\hline 36 & -90 & -79 & -68 & 22 & 74 & -86 & -75.5 & -65 & 21 \\
\hline 38 & -93 & -81.5 & -70 & 23 & & & & & \\
\hline
\end{tabular}




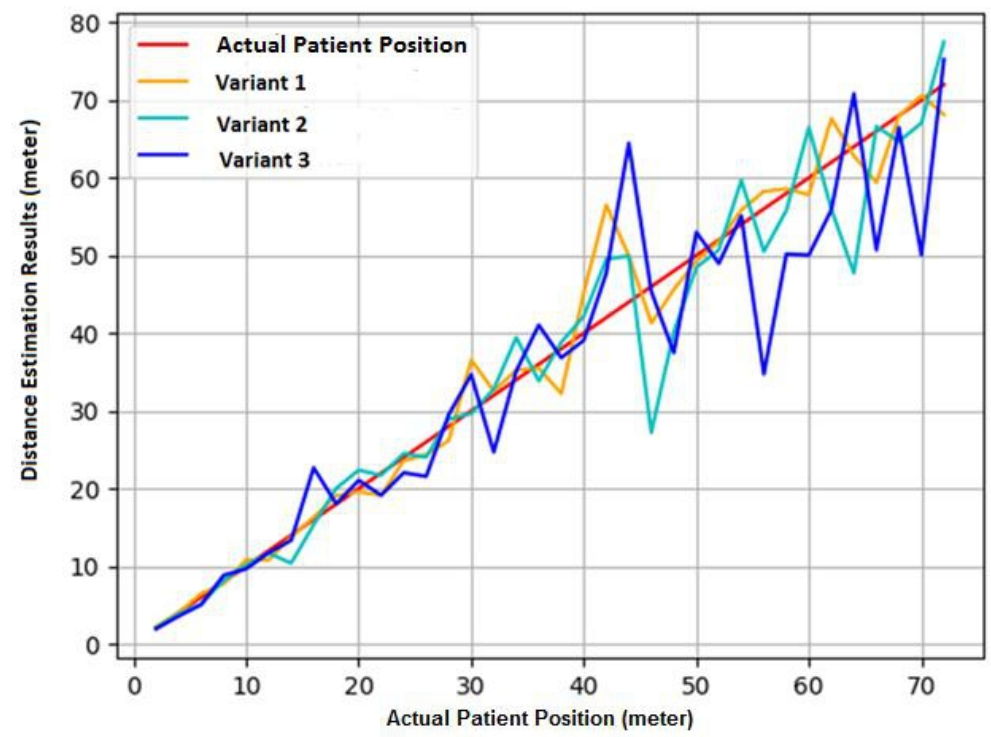

Figure 7 Kalman estimation with a different range.

\section{Testing of The Computational Time of Kalman Filter}

In this test, 4 times computational time test is required for the patient position estimation process. Table 5 shows that the average time required to process Kalman method is $2488.5 \mu$ s. Fig. 8 shows the example of display tracking system. The display obtained aims to test the reliability of the tracking system of Alzheimer's patients, where the blue and green circles are the coverage area of 2 iBeacon patients. The results show that the proposed tracking system able to estimate the position of 2 patients Alzheimer's and displays its position into the form of two circles are blue and green.

Table 5 Computational time of Kalman Method

Test Number
Computational Time $(\mu s)$

2472

2097

1958

2628

2672

2365.4 


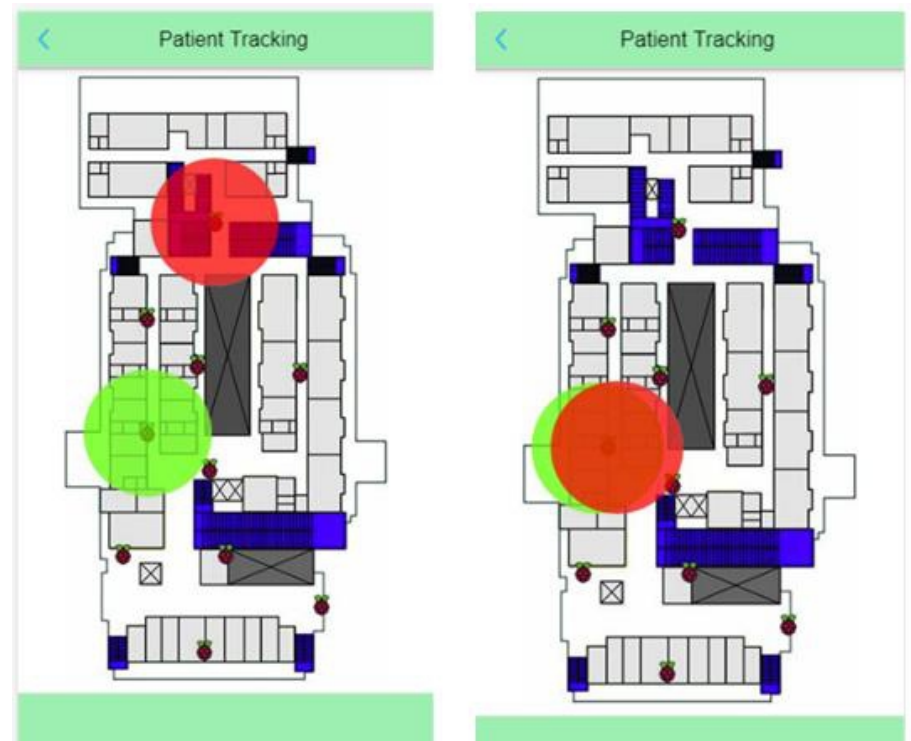

Figure 8 Display of tracking system.

\section{Conclusions}

In this research, we have designed a tracking system for Alzheimer's patients equipped with the Kalman method to estimate the distances. As known RSSI value is strongly influenced by environmental conditions that lead to the acquisition of position estimation is inaccurate. The test results show that the optimum Kalman estimation value obtained when the value of $\mathrm{R}=0.01$ and $\mathrm{Q}=0.1$ with the average percentage error measurement is $7.01 \%$ of the actual patient position. The test results with various data variations also show the reliability of the Kalman method, because the average estimated data approach the actual patient position.

\section{Acknowledgment}

This research was supported by Ministry of Research, Technology, and Higher Education through Penelitian Terapan Unggulan Perguruan Tinggi (PTUPT) Scheme 2017.

\section{References:}

1. Ali, N.H. \& Hassan, G.M., Kalman Filter Tracking, Int.J. of Computer Applications, vol. 89, no.9, pp.15-18,2014.

2. Chancellor, B., Duncan, A. \& Chatterjee, A., Art therapy for Alzheimer's disease and other dementias, J. Alzheimers Dis. JAD, vol. 39, no. 1, pp. 1-11, 2014.

3. Chauhan, P. \& Ahlawat, P., Target Tracking in Wireless Sensor Network, International Journal of Information \& Computation Technology, Vol 4, no 6, pp. 643-648, 2014. 
4. Kristalina, P., Wirawan \& Hendrantoro, G, DOLLY: An experimental evaluation of distributed node positioning framework in wireless sensor networks, in 2014 IEEE Ninth International Conference on Intelligent Sensors, Sensor Networks and Information Processing (ISSNIP), 2014, pp. 1-6.

5. Martin, P., An iBeacon primer for indoor localization demo abstract, Proceedings of the 1st ACM Conference on Embedded Systems for Energy-Efficient Buildings, ACM, 2014.

6. Nebe S.U., Pathloss Prediction Model of a Wireless Sensor Network in an Indoor Environment, IJAREEIE, September 2014.

7. Okumbor, N., Anthony \& Raphael, Characterization of Signal Attenuation using Pathloss Exponent in South-South Nigeria, International Journal of Emerging Trends \& Technology in Computer Science ( IJETTCS), Volume 3, Issue 3, May - June 2014.

8. Pratiarso, A. \& Kristalina, P., An Adaptive Connectivity-based Centroid Algorithm for Node Positioning in Wireless Sensor Networks, Emit. Int. J. Eng. Technol., vol. 3, no. 1, pp. 153 - 163, 2015. 